\title{
Trauma Training Courses and Programs in Low- and Lower Middle-Income Countries: A Scoping Review
}

\author{
Rachel J. Livergant ${ }^{1}$ (i) $\cdot$ Selina Demetrick ${ }^{1} \cdot$ Xenia Cravetchi $^{1} \cdot$ Janice Y. Kung $^{2} \cdot$ Emilie Joos $^{3}$ • \\ Harvey G. Hawes ${ }^{3} \cdot$ Abdullah Saleh $^{1}$
}

\begin{abstract}
Background Injury is the leading cause of morbidity and mortality in low- and lower middle-income countries (LMICs). Trauma training is a cost-effective way to improve injury outcomes. Several trauma programs have been implemented in LMICs; however, their scope and effectiveness remain unclear. In this review, we sought to describe and assess the current state of trauma training in LMICs.

Methods We searched MEDLINE, Embase, Global Health, Cochrane Library, and ProQuest Dissertations \& Theses Global for trauma training courses in LMICs. An additional gray literature search was conducted on university, governmental, and non- governmental organizations' websites to identify trauma-related postgraduate medical education (PGME) opportunities.

Results Most studies occurred in sub-Saharan Africa and participants were primarily physicians/surgeons, medical students/residents, and nurses. General and surgical trauma management courses were most common, followed by orthopedic trauma or plastic surgery trauma/burn care courses. 32/45 studies reported on participant knowledge and skills, 27 of which had minimal follow-up. Of the four studies commenting on cost of courses, only one demonstrated cost-effectiveness. Three articles evaluated post-course effects on patient outcomes, two of which failed to demonstrate significant improvements. Overall, $43.0 \%$ of LMICs have PGME programs with defined trauma competency requirements.

Conclusions Current studies on trauma training in LMICs do not clearly demonstrate sustainability, cost-effectiveness, nor improved outcomes. Trauma training programs should be in response to a need, championed locally, and work within a cohesive system to demonstrate concrete benefits. We recommend standardized and contextualized trauma training with recertifications in LMICs for lasting and improved trauma care.
\end{abstract}

Abdullah Saleh

assaleh@ualberta.ca

1 Office of Global Surgery, Department of Surgery, University of Alberta, Walter Mackenzie Health Sciences Centre, 2D2.238440 - 112 Ave NW, Edmonton, AB T6G 2B7, Canada

2 John W. Scott Health Sciences Library, University of Alberta, Walter C. Mackenzie Health Sciences Centre, 2K3.288440 - 112 Ave NW, Edmonton, AB T6G 2R7, Canada
Division of General Surgery, Trauma and Acute Care Surgery, Vancouver General Hospital, University of British Columbia, Jim Pattison Pavilion, 899 W 12th Ave, Vancouver, BC V5Z 1M9, Canada 


\section{Introduction}

Trauma and injury represent one of the greatest causes of mortality and morbidity globally, more than tuberculosis, HIV and malaria combined $[1,2]$. Despite a decreasing trend in injury-linked disability-associated life years (DALYs) worldwide, low- and lower middle-income countries (LMICs) continue to shoulder a disproportionate burden of traumatic incidents.

Limitations to appropriate trauma care and management in LMICs are multifactorial. After a lack of equipment, inadequate training and education of healthcare providers (HCPs) are the most common barrier to effective trauma care in these settings [3]. Several trauma courses have been implemented in LMICs including Advanced Trauma Life Support (ATLS); Primary Trauma Care (PTC); and Trauma Team Training (TTT).

ATLS is considered the gold standard for trauma training globally; however, the program has faced criticism for being too costly and uncontextualized for low-resource settings $[4,5]$. It was due to the lack of accessibility of ATLS that courses like PTC and TTT emerged. Although PTC and TTT train multidisciplinary teams, unlike ATLS which centers around surgical staff, these courses are narrower in their scope of traumatology topics. Consequently, although these courses have addressed some of the criticism faced by ATLS, there is still a debate as to their effectiveness to meaningfully reduce injury-related morbidity and mortality $[6,7]$.

Another layer of trauma care education in LMICs is postgraduate medical education (PGME) programs, many with specific trauma competencies built into the curricula $[8,9]$. The restructuring and increased development of these PGME programs in LMICs are promising for the field of traumatology; however, there lacks a description of available PGME programs, limiting the ability to comprehensively evaluate trauma training systems in LMICs.

To date, there remains uncertainty as to the extent and effectiveness of trauma training in LMICs. Notably, there is no consensus as to what is the most successful, appropriate, and cost-effective program for definitive hospital care trauma training. This scoping review aims to examine the current ecosystem of trauma training in LMICs, with a specific emphasis on the effectiveness, breadth, multi-disciplinarity, and sustainability of these programs.

\section{Methods}

This scoping review was registered in Open Science Framework (OSF, osf.io/2q9d3) and reported in accordance with Preferred Reporting Items for Systematic
Review and Meta-Analysis-Extension for Scoping Reviews (PRISMA-ScR) framework (Online Resource 1) [10].

\section{Search strategy}

Comprehensive searches were undertaken in Ovid MEDLINE, Ovid Embase, Ovid Global Health, Cochrane Library, and ProQuest Dissertations \& Theses Global on Aug 24, 2020, using relevant keywords (Online Resource 2). Since there were major developments in global trauma training over the last 20 years, the searches were limited to articles published from 2000 and onward [11]. Gray literature, reference lists of reviews and retrieved articles, and consultations with experts were also conducted to identify additional studies.

\section{Study and program selection}

Two reviewers independently screened titles, abstracts, and full texts. Studies were included if they were experimental or observational studies and excluded if they were book chapters, conference abstracts, or non-peer reviewed. Articles pertaining to HCPs, including but not limited to physicians, surgeons, medical officers, nurses, and medical students were included, while those describing laypeople or pre-hospital care providers were excluded. All trauma courses training that focused on trauma including the management of specific injuries such as orthopedic injuries, burns, traumatic brain injuries, and craniomaxillofacial injuries were included. Studies reporting educational programs focused exclusively on ultrasound, telemedicine, triage, trauma registries, critical care, and emergency medicine (including obstetrics, neonatology, and infectious diseases) were excluded.

\section{Data extraction and analysis}

The following information was extracted from individual studies using a standardized extraction form: publication year, study setting, characteristics of trainees, course details, and course outcomes. Data from primary studies were extracted by one reviewer and independently verified by a second reviewer. Any discrepancies were resolved by consensus.

Study characteristics were analyzed and presented using a mixed-methods approach. The heterogeneous nature of this review precluded a meta-analysis. Instead, a narrative synthesis of results was undertaken using evidence tables and figures to aid in data presentation, when appropriate.

Outcomes included course effectiveness, suitability, sustainability, and breadth of topics and training. Course effectiveness was defined based on changes to patient 
morbidity and mortality or skills of participants. Suitability refers to the extent to which courses were contextualized to the setting and engaged local stakeholders during implementation. Sustainability reflects the longevity of the course in terms of follow-up, recertification processes and cost-effectiveness, while course breadth refers to the variety of types of HCP participants and trauma topics.

\section{Gray literature search for PGME programs}

A gray literature search for surgical PGME programs with trauma components in LMICs was undertaken. Accredited programs such as residency, fellowship, diploma, certificate, Masters, and Doctoral programs were used as search terms, identified, and included for consideration. PGME programs were reviewed for trauma components and duration.

\section{Results}

\section{Trauma care training courses}

We retrieved 4305 articles from the electronic search, of which 3095 underwent title and abstract screening. Seven studies were multiple publications reporting on the same training course or study cohort and therefore are reported with their parent study to avoid duplication, resulting in 45 unique studies (Fig. 1) [12-63]. Descriptions of included studies can be found in Table 1 .

\section{Participants and setting}

The majority of studies reported on multidisciplinary courses $(26 / 45,57.8 \%)$, involving nurses, physicians, residents, medical students, medical/clinical officers, and other allied HCPs. Physicians and surgeons were the most common group of participants $(27 / 45,60.0 \%)$, followed by nurses $(14 / 45,31.1 \%)$, medical students $(12 / 45,26.7 \%)$, and residents $(11 / 45,24.4 \%)$. Other participants included in studies were unspecified HCPs $(6 / 45,13.3 \%)$, medical and/or clinical officers $(5 / 45,11.1 \%)$, rehabilitation therapists/physiotherapists $(2 / 45,4.4 \%)$, village health workers $(1 / 45,2.2 \%)$, emergency medical technicians $(1 / 45,2.2 \%)$, and/or midwives $(1 / 45,2.2 \%)$.

Included articles spanned 27 LMICs, with most of the studies $(21 / 45,46.7 \%)$ occurring in sub-Saharan Africa [38-63]. Nearly all of the studies were conducted in an urban setting $(34 / 45,75.6 \%)$, with only seven $(15.6 \%)$ describing a rural-specific program $[12,13,15,31,46,49,62]$ and four studies (8.9\%) defining training in both urban and rural settings [17, 32, 41, 45].

\section{Suitability and local engagement}

Most studies engaged local stakeholders (43/45; 95.6\%), in the form of co-creation or implementation of courses with local organizations $(34 / 45 ; 75.6 \%)$; co-authorship including a member from the study setting (37/45; 82.2\%); and/or study conducted by local stakeholders themselves $(9 / 45$; $20.0 \%$ ). However, only 15/45 (33.3\%) studies completed a formal needs assessment before implementing a trauma course. The needs assessments that were done examined factors such as availability of resources and personnel $[14,19,24,25,29,41,44,49,60,62,63]$ local injury burden [24, 44, 60]; current training methods, gaps in training and future training requirements $[14,16,19,25,29,41,46,49,56,60,63]$; cost and suitability of the program $[25,41,56,62]$; and optimal course delivery plans $[16,44,60]$.

\section{Courses breadth and competencies}

Thirty-two different courses were described in retrieved studies, the majority of which focused on general surgical trauma training $(34 / 45,75.6 \%)$, with 14 of these courses being individualized for a specific setting in conjunction with a non-governmental organization (NGO) or high-income university. Of pre-established programs, PTC was the most commonly described course (7/45, 15.6\%) [19, 26, 35, 36, 38, 40, 45], followed by Trauma Evaluation and Management (TEAM) (4/45, 8.9\%) [23, 37, 48, 50], TTT $(3 / 45,6.7 \%)[52,54,56]$, and ATLS $(3 / 45,6.7 \%)$ [14, 30, 52].

Orthopedic trauma injury management was the focus of six $(13.3 \%)$ studies [4, 18, 21, 22, 55, 57], while burn management was the focus of four, of which two reported on the implementation of the American Burn Association/ Children's Burn Foundation (ABA/CBF) Team Training program [29, 62]. Only one study reported on trauma related to plastic surgery reconstruction [44].

\section{Course effectiveness}

Most studies found an improvement in trauma management skills and knowledge, regardless of course type (32/45, $71.1 \%$ ). Most of the studies reported increase in participant skills and/or knowledge of trauma management based on pre-course versus post-course tests and surveys (28/45, $62.2 \%$ ), while the remaining four studies evaluated knowledge through self-reflection surveys or interviews.

Twelve (26.7\%) studies commented on changes to trauma management practices and patient outcomes post-course, of which seven had data on pre-course versus post-course outcomes [13, 19, 21, 29, 38, 46, 51, 52, 55, 58, 60, 62]. Only three studies objectively evaluated pre- to post-course 


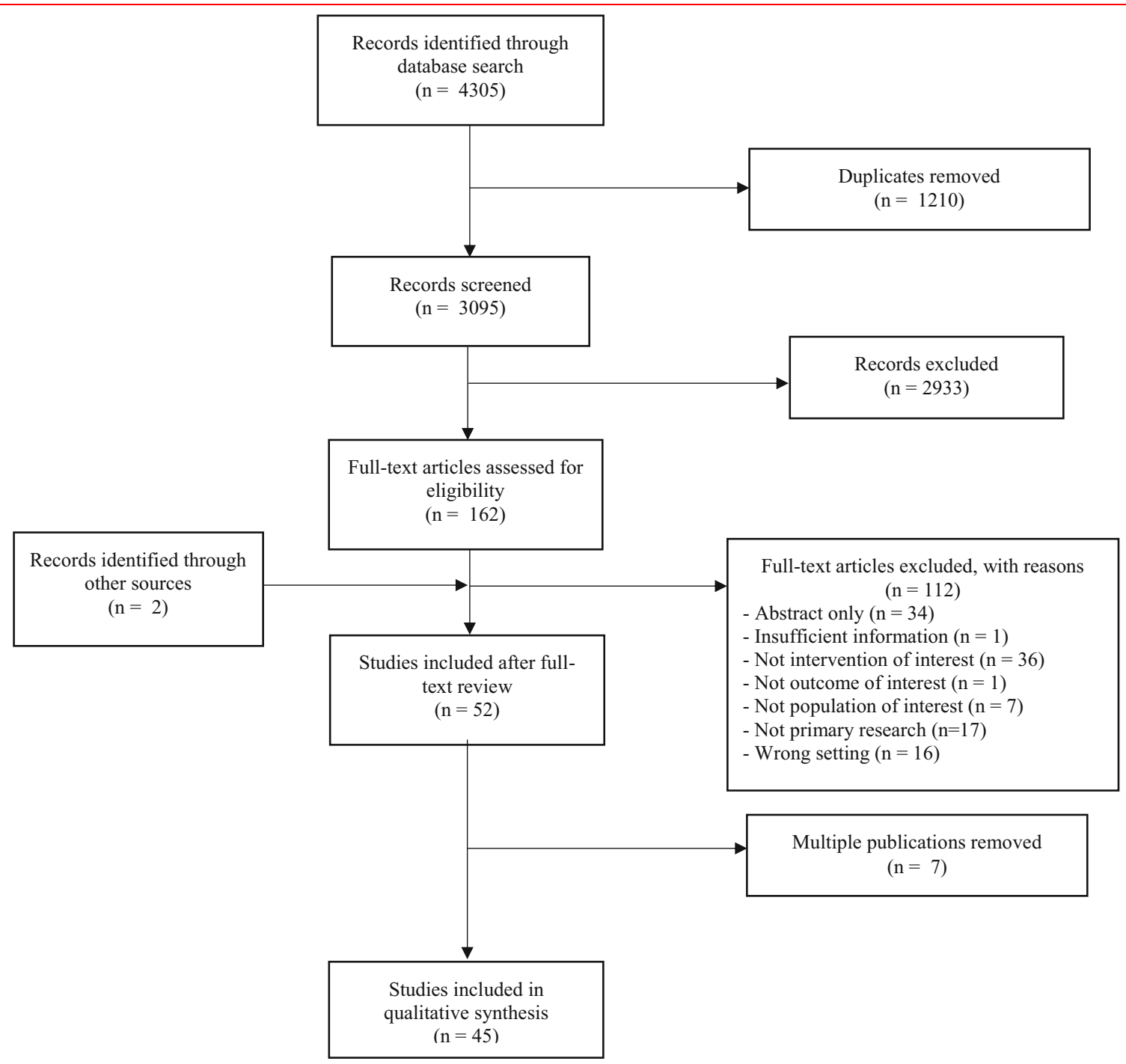

Fig. 1 The literature search and study selection flowchart

changes in patient mortality and morbidity [19, 52, 62], of which two failed to find significant improvements [19, 62].

Cioè-Peña et al. [19] reported a significant increase in the use of FAST exams; however, other facets of trauma management, including critical skills and time to operating room, were not significantly altered by PTC training. Ullrich et al. [60] found an improvement from pre-course to post-course test scores, but actual trauma operative management techniques did not significantly improve.

Two studies implemented and examined $\mathrm{ABA} / \mathrm{CBF}$ training on burn management competencies and patient outcomes [29, 62]. One study in India reported improved burn management skills, including decreased antibiotic use and earlier excision and grafting [29], while a similar study in Zambia did not report significant changes to burn management protocols or consequent patient outcomes [62].
Carey et al. [55] reported on the completion of 93.3\% flaps with a $78.1 \%$ aversion rate of amputations, while Tajsic et al. [13] indicated a 70\% primary healing rate of open fractures after orthopedic trauma courses. Participants of the Uganda Sustainable Trauma Orthopedic Program reported a subjective improvement for patients with orthopedic trauma [58]. Additionally, the orthopedic trauma PGME program in Malawi and Haiti demonstrated a significant improvement in patient outcomes post-training [21, 51].

ATLS training significantly decreased mortality of severely injured patients in Rwanda [52]. PTC training in El Salvador, and ABA/CBF training in Zambia did not result in improved patient mortality rates post-training $[19,62]$. 
Table 1 Description of trauma training courses in low- and lower middle-income countries identified from electronic database search

\begin{tabular}{|c|c|c|c|c|}
\hline $\begin{array}{l}\text { First author, } \\
\text { year }\end{array}$ & Country & Program & Participants (n) & Outcome(s) \\
\hline \multicolumn{5}{|c|}{ East Asia and Pacific } \\
\hline $\begin{array}{l}\text { Van Heng, } \\
2008[12]\end{array}$ & Cambodia & $\begin{array}{l}\text { "Surgical trauma training for non- } \\
\text { surgeons" in rural hospitals }\end{array}$ & $\begin{array}{l}\text { Physicians (2) } \\
\text { Medical officers } \\
\text { (19) }\end{array}$ & $\begin{array}{l}\text { Increased overall confidence and } \\
\text { skills managing trauma after } \\
\text { training compared to before } \\
\text { training based on scoring from } \\
\text { instructors }\end{array}$ \\
\hline $\begin{array}{l}\text { Tajsic, } 2017 \\
\text { [13] }\end{array}$ & Cambodia & $\begin{array}{l}\text { Open Fracture Training Course } \\
\text { (including post-traumatic defects) }\end{array}$ & Surgeons (112) & $\begin{array}{l}\text { Significant improvement of } \\
\text { theoretical understanding, } \\
\text { technical skills and self-confidence } \\
\text { in independent management of } \\
\text { open fractures and reported a } 70 \% \\
\text { primary healing rate of fractures }\end{array}$ \\
\hline $\begin{array}{l}\text { Kornfeld, } \\
2019[14]\end{array}$ & Mongolia & $\begin{array}{l}\text { Advanced Trauma Life Support } \\
\text { (ATLS) }\end{array}$ & Physicians & $\begin{array}{l}\text { Annual cost of ATLS with } \\
\text { Mongolian instructors would be } \\
\text { USD 10,709 compared to USD } \\
68,038 \text { if foreign instructors are } \\
\text { required every year }\end{array}$ \\
\hline $\begin{array}{l}\text { Richard, } \\
2009[15]\end{array}$ & Myanmar & $\begin{array}{l}\text { Trauma Management Program } \\
\text { (TMP) }\end{array}$ & $\begin{array}{l}\text { Village health } \\
\text { workers }(300)\end{array}$ & $\begin{array}{l}\text { Majority of patients treated by } \\
\text { trainees survived (91\%). } \\
\text { Unstructured interviews revealed } \\
\text { that training during workshops was } \\
\text { utilized in the field for trauma care }\end{array}$ \\
\hline $\begin{array}{l}\text { Yu, } 2017 \\
{[16]}\end{array}$ & Solomon Islands & Pediatric Acute Care Training Course & Physicians (57) & $\begin{array}{l}\text { Participants reported a mean } 4.81 / 5 \\
\text { overall satisfaction with the } \\
\text { course, but the course needed } \\
\text { further sociocultural modifications. } \\
\text { Post-course test results increased } \\
\text { from } 69.6 \% \text { to } 77.6 \%\end{array}$ \\
\hline $\begin{array}{l}\text { Lam, } 2018 \\
\quad[17]\end{array}$ & Vietnam & Burn Management Training & $\begin{array}{l}\text { Physicians (174) } \\
\text { Nurses (131) }\end{array}$ & $\begin{array}{l}\text { Participants significantly increased } \\
\text { their knowledge scores on burn } \\
\text { emergency management } \\
(\mathrm{p}<0.01) \text { and mass burn injury } \\
\text { management }(\mathrm{p}<0.001) \text { after } \\
\text { participation in the course }\end{array}$ \\
\hline $\begin{array}{l}\text { Choi, } 2020 \\
\quad[18]\end{array}$ & Vietnam & $\begin{array}{l}\text { Orthopedic Trauma Care Capacity } \\
\text { Strengthening Training Workshop }\end{array}$ & $\begin{array}{l}\text { Surgeons (19) } \\
\text { Rehabilitation } \\
\text { therapists (6) } \\
\text { Nurses (2) }\end{array}$ & $\begin{array}{l}\text { The orthopedic training fellowship } \\
\text { workshop on trauma care received } \\
\text { high satisfaction ratings from } \\
\text { participants in terms of satisfaction } \\
\text { of teaching tools }(4.28 / 5) \text {; } \\
\text { perceived benefit of course }(4.17 / \\
\text { 5) and overall quality of the } \\
\text { workshop (4.32/5) }\end{array}$ \\
\hline \multicolumn{5}{|c|}{ Latin America \& Caribbean } \\
\hline $\begin{array}{l}\text { Cioè-Peña, } \\
2017 \text { and } \\
\text { Dickason, } \\
2017 \\
{[19,20]}\end{array}$ & El Salvador & Primary Trauma Care (PTC) & $\begin{array}{l}\text { Physicians and } \\
\text { nurses (18) }\end{array}$ & $\begin{array}{l}\text { Overall critical skills in trauma } \\
\text { management, time to operating } \\
\text { room and mortality rates of trauma } \\
\text { patients were not significantly } \\
\text { altered by PTC. Number of FAST } \\
\text { exams increased from } 9.5 \% \text { (pre- } \\
\text { training) to } 21.4 \% \text { post-training } \\
(\mathrm{p}=0.04)\end{array}$ \\
\hline $\begin{array}{l}\text { Carlson, } \\
2012[21]\end{array}$ & Haiti & $\begin{array}{l}\text { Orthopedic Trauma Care Specialist } \\
\text { (OTCS) Residency Program }\end{array}$ & Surgical residents & $\begin{array}{l}\text { Orthopedic trauma care specialists } \\
\text { care versus traditional care } \\
\text { resulted in } 12,213 \text { DALYs averted } \\
\text { and cost savings of USD } \\
\text { 134/DALY for every resident } \\
\text { trained }\end{array}$ \\
\hline
\end{tabular}


Table 1 continued

\begin{tabular}{|c|c|c|c|c|}
\hline $\begin{array}{l}\text { First author, } \\
\text { year }\end{array}$ & Country & Program & Participants (n) & Outcome(s) \\
\hline $\begin{array}{l}\text { Normore, } \\
2017 \text { [22] }\end{array}$ & Haiti & $\begin{array}{l}\text { Orthopedic Trauma Symposium } \\
\text { (OTS) }\end{array}$ & $\begin{array}{l}\text { Medical students. } \\
\quad(29) \\
\text { Residents (21) } \\
\text { Physicians (9) } \\
\text { Other (11) }\end{array}$ & $\begin{array}{l}\text { Participants were satisfied with the } \\
\text { level of difficulty (3.59), } \\
\text { organization of topics ( } 4.09) \text {, } \\
\text { usefulness of exercises and } \\
\text { helpfulness of instructional aids } \\
\text { (4.71) of the OTS based on a five- } \\
\text { point Likert scale }\end{array}$ \\
\hline $\begin{array}{l}\text { Kurdin, } 2018 \\
\text { [23] }\end{array}$ & Haiti & $\begin{array}{l}\text { Trauma Evaluation and Management } \\
\text { (TEAM) }\end{array}$ & $\begin{array}{l}\text { Physicians (32) } \\
\text { Nurses (22) } \\
\text { EMTs (10) } \\
\text { Medical students } \\
\quad(5)\end{array}$ & $\begin{array}{l}\text { Survey responses indicated that the } \\
\text { course was satisfactory but needed } \\
\text { adjustments for LMICs to include } \\
\text { unavailability of specialists and } \\
\text { increased nursing responsibilities }\end{array}$ \\
\hline $\begin{array}{l}\text { Pringle, } \\
2015[24]\end{array}$ & Nicaragua & $\begin{array}{l}\text { "Short trauma course with low cost } \\
\text { simulation" }\end{array}$ & $\begin{array}{l}\text { Physicians (18) } \\
\text { Residents (15) }\end{array}$ & $\begin{array}{l}\text { Written simulation scores improved } \\
\text { from pre- to post- course (test } \\
\text { increase by } 15.4 \%, \mathrm{p}<0.001 \text { and } \\
\text { simulation by } 33.6 \%, \mathrm{p}<0.001 \text { ). } \\
\text { Primary survey and c-spine } \\
\text { immobilization times during } \\
\text { simulation also improved }\end{array}$ \\
\hline \multicolumn{5}{|c|}{ Middle East \& North Africa } \\
\hline $\begin{array}{l}\text { El-Shinawi, } \\
2015[25]\end{array}$ & Egypt & $\begin{array}{l}\text { Sequential Trauma Emergency/ } \\
\text { Education ProgramS (STEPS) }\end{array}$ & $\begin{array}{l}\text { Physicians, } \\
\text { Surgeons, } \\
\text { Residents, } \\
\text { Laypeople (639) }\end{array}$ & $\begin{array}{l}\text { Course demonstrated the } \\
\text { sustainability of a trauma course } \\
\text { transitioned from a high-income } \\
\text { country to the LMIC over seven } \\
\text { years }\end{array}$ \\
\hline $\begin{array}{l}\text { Alwawi, } \\
2019[26]\end{array}$ & West Bank & Primary Trauma Care (PTC) & $\begin{array}{l}\text { Physicians and } \\
\text { Medical } \\
\text { Students (445) } \\
\text { Nurses (130) } \\
\text { Midwives (11) }\end{array}$ & $\begin{array}{l}\text { Increased knowledge and } \\
\text { understanding of trauma } \\
\text { management skills post-training } \\
(15.76 / 20) \text { compared to pre- } \\
\text { training }(9.43 / 20)(\mathrm{p}<0.001)\end{array}$ \\
\hline \multicolumn{5}{|l|}{ South Asia } \\
\hline $\begin{array}{l}\text { Biswas, } \\
2017[27]\end{array}$ & Bangladesh & $\begin{array}{l}\text { Emergency Management of Severe } \\
\text { Burns (EMSB) }\end{array}$ & Physicians (38) & $\begin{array}{l}\text { Self-reported surveys indicate } 43.8 \% \\
\text { and } 31.2 \% \text { of participants felt more } \\
\text { confident in their overall burn } \\
\text { management skills and handling of } \\
\text { critical care burns, respectively }\end{array}$ \\
\hline $\begin{array}{l}\text { Tchorz, } 2007 \\
\text { [28] }\end{array}$ & India & "Intensive trauma course" & $\begin{array}{l}\text { Residents (23) } \\
\text { Physicians and } \\
\text { Surgeons (9) }\end{array}$ & $\begin{array}{l}\text { Participants had significantly } \\
\text { improved mean scores from pre- } \\
\text { course }(70.7 \%) \text { to post-course } \\
(87.5 \%) \text { trauma management } \\
\text { knowledge tests }(\mathrm{p}<0.001)\end{array}$ \\
\hline $\begin{array}{l}\text { Light, } 2009 \\
\text { [29] }\end{array}$ & India & $\begin{array}{l}\text { American Burn Association/ } \\
\text { Children's Burn Foundation } \\
\text { (ABA/CBF) Team Training }\end{array}$ & $\begin{array}{l}\text { Healthcare } \\
\text { practitioners } \\
(60)\end{array}$ & $\begin{array}{l}\text { Study reports improved nursing } \\
\text { satisfactions, patient tolerance, } \\
\text { burn management knowledge, } \\
\text { earlier excision, and grafting and } \\
\text { decreased antibiotic use post- } \\
\text { intervention }\end{array}$ \\
\hline $\begin{array}{l}\text { Douglas, } \\
2010[30]\end{array}$ & India & $\begin{array}{l}\text { Advanced Trauma Life Support } \\
\text { (ATLS) }\end{array}$ & $\begin{array}{l}\text { Medical officers } \\
\quad \text { (Indian = 77; } \\
\quad \text { Australian }=44)\end{array}$ & $\begin{array}{l}\text { Trauma knowledge quiz scores } \\
\text { demonstrated that the net pass rate } \\
\text { for those who completed ATLS } \\
\text { was } 97 \% \text { compared to } 15 \% \text { for } \\
\text { those who did not take ATLS }\end{array}$ \\
\hline
\end{tabular}


Table 1 continued

\begin{tabular}{|c|c|c|c|c|}
\hline $\begin{array}{l}\text { First author, } \\
\text { year }\end{array}$ & Country & Program & Participants (n) & Outcome(s) \\
\hline $\begin{array}{l}\text { Ali, } 2015 \\
\text { [31] }\end{array}$ & India & $\begin{array}{l}\text { Rural Trauma Team Development } \\
\text { Course (RTTDC) }\end{array}$ & $\begin{array}{l}\text { Healthcare } \\
\text { providers (23) }\end{array}$ & $\begin{array}{l}\text { Participants improved their trauma } \\
\text { management knowledge and } \\
\text { understanding (pre-test }=6.8 \\
\text { post-test }=13.6, \mathrm{p}<0.001 \text { ) after } \\
\text { the RTTDC. Additionally, the } \\
\text { course had a high participant } \\
\text { satisfaction level }\end{array}$ \\
\hline $\begin{array}{l}\text { O'Reilly, } \\
2009 \text { and } \\
2011 \\
{[32,33]}\end{array}$ & India and Sri Lanka & $\begin{array}{l}\text { Alfred Trauma Team Training } \\
\text { Program }\end{array}$ & $\begin{array}{l}\text { Nurses (14) } \\
\text { Physicians (12) }\end{array}$ & $\begin{array}{l}\text { Participants demonstrated an } \\
\text { improvement on team-based } \\
\text { trauma scenarios, multiple choice } \\
\text { tests, and written evaluations }\end{array}$ \\
\hline $\begin{array}{l}\text { Shrestha, } \\
2018[34]\end{array}$ & Nepal & $\begin{array}{l}\text { Emergency Room Trauma Course } \\
\text { (ERTC) }\end{array}$ & $\begin{array}{l}\text { Medical Students } \\
\quad(97)\end{array}$ & $\begin{array}{l}\text { Participant MCQ knowledge scores } \\
\text { improved from } 64.2 \% \text { to } 89.8 \% \\
\text { (p }<0.001) \text { and skills scores on } \\
\text { OSCEs improved from } 33.2 \% \text { to } \\
78.6 \%(\mathrm{p}<0.001)\end{array}$ \\
\hline $\begin{array}{l}\text { Jawaid, } 2013 \\
\quad[35]\end{array}$ & Pakistan & Primary Trauma Care (PTC) & $\begin{array}{l}\text { Physicians, } \\
\text { Surgeons, } \\
\text { Residents and } \\
\text { Medical } \\
\text { students (20) }\end{array}$ & $\begin{array}{l}\text { Participants demonstrated increased } \\
\text { trauma management skills (pre- } \\
\text { test }=3.5 / 20, \text { post-test }=9.5 / 20, \\
\mathrm{p}<0.0001 \text { ) and knowledge (pre- } \\
\text { test }=19.5 / 30, \text { post-test }=25 / 30, \\
\mathrm{p}<0.0001 \text { ) after the PTC course. } \\
\text { Although } 95 \% \text { of participants } \\
\text { scored }>70 \% \text { on the knowledge } \\
\text { test, only } 20 \% \text { of participants } \\
\text { scored }>70 \% \text { on the skills portion } \\
\text { post-course }\end{array}$ \\
\hline $\begin{array}{l}\text { Sadiq, } 2018 \\
\quad[36]\end{array}$ & Pakistan & Primary Trauma Care (PTC) & $\begin{array}{l}\text { Medical students } \\
\quad(77)\end{array}$ & $\begin{array}{l}\text { Participants scored on average } 5.104 \\
\text { points higher on their post-course } \\
\text { trauma management quizzes } \\
\text { compared to their pre-course } \\
\text { quizzes }(\mathrm{p}<0.001)\end{array}$ \\
\hline $\begin{array}{l}\text { Soomro, } \\
2020 \text { [37] }\end{array}$ & Pakistan & $\begin{array}{l}\text { Trauma Evaluation and Management } \\
\text { (TEAM) }\end{array}$ & $\begin{array}{l}\text { Medical students } \\
\text { (294) }\end{array}$ & $\begin{array}{l}\text { The course had a high satisfaction } \\
\text { rate. With }>85 \% \text { of participants } \\
\text { indicating usefulness of the course } \\
\text { in future practice and }>80 \% \text { of } \\
\text { faculty wishing to be involved in } \\
\text { TEAM instruction. As well, there } \\
\text { was a significant increase in } \\
\text { trauma management knowledge } \\
\text { for groups receiving TEAM } \\
\text { training versus those without the } \\
\text { course }(\mathrm{p}<0.001)\end{array}$ \\
\hline \multicolumn{5}{|c|}{ Sub-Saharan Africa } \\
\hline $\begin{array}{l}\text { Ologunde, } \\
2017 \text { and } \\
\text { Peter, } \\
2015 \\
{[38,39]}\end{array}$ & $\begin{array}{l}\text { Burundi, Ethiopia, Kenya, } \\
\text { Malawi, Mozambique, } \\
\text { Rwanda, Tanzania, } \\
\text { Uganda, Zambia and } \\
\text { Zimbabwe }\end{array}$ & Primary Trauma Care (PTC) & $\begin{array}{l}\text { Physicians (253) } \\
\text { Nurses (98) } \\
\text { Medical students } \\
\quad(44) \\
\text { Clinical officers } \\
\quad(40)\end{array}$ & $\begin{array}{l}\text { Six months after training, } 92.7 \% \text { of } \\
\text { respondents indicated improved } \\
\text { trauma management, with } 52.8 \% \\
\text { using a systematic or ABCDE } \\
\text { approach. Departmental changes to } \\
\text { trauma care were mainly moderate } \\
(30 \%) \text { with } 23 \% \text { of respondents } \\
\text { indicating no change. Only } 24.8 \% \\
\text { of respondents perceived } \\
\text { improvement in trauma patient } \\
\text { mortality and morbidity }\end{array}$ \\
\hline
\end{tabular}


Table 1 continued

\begin{tabular}{|c|c|c|c|c|}
\hline $\begin{array}{l}\text { First author, } \\
\text { year }\end{array}$ & Country & Program & Participants (n) & Outcome(s) \\
\hline $\begin{array}{l}\text { Nogaro, } \\
2015[40]\end{array}$ & $\begin{array}{l}\text { Ethiopia, Kenya, Malawi, } \\
\text { Mozambique, Rwanda, } \\
\text { Uganda, Zimbabwe }\end{array}$ & Primary Trauma Care (PTC) & $\begin{array}{l}\text { Physicians and } \\
\text { Surgeons (240) } \\
\text { Other healthcare } \\
\text { practitioners } \\
(105)\end{array}$ & $\begin{array}{l}91 \% \text { of candidates showed a } \\
\text { significant improvement in } \\
\text { knowledge after the PTC course, } \\
\text { with a median improvement of } \\
17 \%(\mathrm{p}<0.05) \text {. As well, } \\
\text { candidates' confidence in trauma } \\
\text { management improved by } 20 \%\end{array}$ \\
\hline $\begin{array}{l}\text { MacLeod, } \\
2009 \text { and } \\
2011 \\
{[41,42]}\end{array}$ & Kenya, Zambia & $\begin{array}{l}\text { Acute Trauma Care and Fundamental } \\
\text { Critical Care Support Course } \\
\text { (ATC/FCCS) }\end{array}$ & $\begin{array}{l}\text { Medical officers } \\
\text { (27) } \\
\text { Physicians and } \\
\text { Surgeons (21) } \\
\text { Nurses (14) } \\
\text { Clinical officers } \\
\text { (13) }\end{array}$ & $\begin{array}{l}\text { Participants had increased overall } \\
\text { trauma knowledge from an average } \\
\text { of } 51 \% \text { to } 63 \%(\mathrm{p}=0.002) \text { post- } \\
\text { course. Additionally, participants } \\
\text { reported increased confidence over } \\
\text { all } 22 \text { presented clinical trauma } \\
\text { scenarios and } 15 \text { procedures }\end{array}$ \\
\hline $\begin{array}{l}\text { O’Sullivan, } \\
2012[43]\end{array}$ & Malawi, Tanzania, Zambia & $\begin{array}{l}\text { Global Emergency Care Skills } \\
\text { (GECS) }\end{array}$ & $\begin{array}{l}\text { Physicians (80) } \\
\text { Nurses (5) } \\
\text { Clinical officers } \\
\text { (2) }\end{array}$ & $\begin{array}{l}\text { Trauma management knowledge test } \\
\text { scores were significantly improved } \\
\text { from pre-course }(58.71 \%) \text { to post- } \\
\text { course }(78.26 \%)(\mathrm{p}=0.0001) \text { for } \\
\text { participants from all countries }\end{array}$ \\
\hline $\begin{array}{l}\text { Shaye, } 2018 \\
\text { [44] }\end{array}$ & Rwanda, Zimbabwe & $\begin{array}{l}\text { Craniomaxillofacial Trauma Course } \\
\text { "Essentials in Facial Injuries" } \\
\text { Course }\end{array}$ & $\begin{array}{l}\text { Residents (12) } \\
\text { Surgeons (5) } \\
\text { Unknown/Other } \\
\quad(6)\end{array}$ & $\begin{array}{l}\text { Mean pre-course and post-course test } \\
\text { scores increased in both cohorts } \\
\text { (cohort } 1=30 \% \text { increase, cohort } \\
2=12 \% \text { increase) }\end{array}$ \\
\hline $\begin{array}{l}\text { Tolppa, } 2020 \\
{[45]}\end{array}$ & $\begin{array}{l}\text { Democratic Republic of } \\
\text { the Congo }\end{array}$ & Primary Trauma Care (PTC) & $\begin{array}{l}\text { Physicians (36) } \\
\text { and Nurses (23) }\end{array}$ & $\begin{array}{l}\text { Although increased post-course test } \\
\text { scores were maintained over two } \\
\text { years, confidence skills } \\
\text { significantly decreased ( } \mathrm{p}=0.03) \text {. } \\
\text { As well, } 36 \text { participants indicated a } \\
\text { lack of equipment availability, } \\
\text { while } 52 \text { felt different procedures } \\
\text { were required for managing local } \\
\text { patients }\end{array}$ \\
\hline $\begin{array}{l}\text { Mock, } 2005 \\
\text { and } \\
\text { Quansah, } \\
2008 \\
{[46,47]}\end{array}$ & Ghana & $\begin{array}{l}\text { Kwame Nkrumah University of } \\
\text { Science and Technology (KNUST) } \\
\text { Trauma Course for Rural Hospitals }\end{array}$ & Physicians (83) & $\begin{array}{l}\text { Participants had significant increases } \\
\text { in post-course scores and the } \\
\text { majority felt trauma care practice } \\
\text { had improved at one-year follow- } \\
\text { up. Recommendations for } \\
\text { improvement included more } \\
\text { practical sessions, focus on } \\
\text { orthopedics, and longer course } \\
\text { duration }\end{array}$ \\
\hline $\begin{array}{l}\text { Berndtson, } \\
2019[48]\end{array}$ & Ghana & $\begin{array}{l}\text { Trauma Evaluation and Management } \\
\text { (TEAM) }\end{array}$ & $\begin{array}{l}\text { Medical students } \\
\quad(62)\end{array}$ & $\begin{array}{l}\text { TEAM training resulted in higher } \\
\text { post-course scores }(69.1 \%) \\
\text { compared to pre-course }(44.2 \%) \\
\text { scores }(\mathrm{p}<0.001) \text {. Ghanaian } \\
\text { medical students indicated } \\
\text { infrastructure, teamwork issues } \\
\text { and lack of physical equipment as } \\
\text { major barriers to trauma care }\end{array}$ \\
\hline $\begin{array}{l}\text { Wanjiku, } \\
2017[49]\end{array}$ & Kenya & "Emergency trauma course" & $\begin{array}{l}\text { Medical students } \\
\quad(22)\end{array}$ & $\begin{array}{l}\text { Immediately post-course, test scores } \\
\text { and simulation scores increased by } \\
\text { a mean of } 15.5 \% \text { and } 45.5 \%, \\
\text { respectively }(\mathrm{p}=0.0001) \text {. At nine } \\
\text { months follow-up, simulation, } \\
\text { knowledge and confidence scores } \\
\text { had not significantly changed }\end{array}$ \\
\hline
\end{tabular}


Table 1 continued

\begin{tabular}{|c|c|c|c|c|}
\hline $\begin{array}{l}\text { First author, } \\
\text { year }\end{array}$ & Country & Program & Participants (n) & Outcome(s) \\
\hline $\begin{array}{l}\text { Hill, } 2018 \\
\quad[50]\end{array}$ & Kenya & $\begin{array}{l}\text { Trauma Evaluation and Management } \\
\text { (TEAM) }\end{array}$ & $\begin{array}{l}\text { Medical students } \\
\text { (61) }\end{array}$ & $\begin{array}{l}\text { TEAM training resulted in higher } \\
\text { post-course scores }(72 \%) \\
\text { compared to pre-course }(57 \%) \\
\text { scores }(\mathrm{p}<0.001)\end{array}$ \\
\hline $\begin{array}{l}\text { Young, } 2016 \\
{[51]}\end{array}$ & Malawi & $\begin{array}{l}\text { Postgraduate Medical Education for } \\
\text { Surgical Trauma }\end{array}$ & Residents (12) & $\begin{array}{l}\text { The study reported on the creation of } \\
\text { cohesive, national surgical } \\
\text { residency programs with training } \\
\text { in trauma care. Amputations } \\
\text { significantly decreased in this } \\
\text { period, while limb saving surgeries } \\
\text { increased }\end{array}$ \\
\hline $\begin{array}{l}\text { Petroze, } \\
\quad 2015[52]\end{array}$ & Rwanda & $\begin{array}{l}\text { Advanced Trauma Life Support } \\
\text { (ATLS) and Trauma Team } \\
\text { Training (TTT) }\end{array}$ & $\begin{array}{l}\text { Surgeons, Nurses, } \\
\text { Residents (64) }\end{array}$ & $\begin{array}{l}\text { There was a significant reduction in } \\
\text { mortality rate of severely injured } \\
\text { patients, but no significant } \\
\text { difference in mortality rate of the } \\
\text { entire patient population or } \\
\text { resource utilization six months } \\
\text { post-training }\end{array}$ \\
\hline $\begin{array}{l}\text { Bergman } \\
\quad 2008[53]\end{array}$ & Tanzania & Trauma Team Training (TTT) & $\begin{array}{l}\text { Nurses (13) } \\
\text { Physicians (7) }\end{array}$ & $\begin{array}{l}\text { Median post-course test scores }(13 / \\
15) \text { increased significantly } \\
\text { compared to pre-course }(9 / 15) \\
\text { knowledge of trauma management } \\
(\mathrm{p}=0.0004)\end{array}$ \\
\hline $\begin{array}{l}\text { Mitchell, } \\
\quad 2013[54]\end{array}$ & Tanzania & Essential Surgical Skills (ESS) & $\begin{array}{l}\text { Medical students } \\
\quad(60)\end{array}$ & $\begin{array}{l}\text { The study describes increased } \\
\text { technical trauma surgical skills and } \\
\text { confidence post-workshop }\end{array}$ \\
\hline $\begin{array}{l}\text { Carey, } 2015 \\
\text { [55] }\end{array}$ & Tanzania & $\begin{array}{l}\text { Surgical Management and } \\
\text { Reconstructive Training course } \\
\text { (SMART) for Orthopedic Trauma }\end{array}$ & Surgeons (34) & $\begin{array}{l}\text { The SMART course resulted in } \\
93.3 \% \text { (554) successful flap } \\
\text { procedures and aversion of } 78.1 \% \\
\text { of potential amputations. Overall, } \\
\text { the course cost USD } \\
18,000-25,000\end{array}$ \\
\hline $\begin{array}{l}\text { Lett, } 2004 \\
\quad[56]\end{array}$ & Uganda & Trauma Team Training (TTT) & $\begin{array}{l}\text { Healthcare } \\
\quad \text { Professionals }\end{array}$ & $\begin{array}{l}\text { All trainees noted gained practical } \\
\text { skills and trauma teamwork and } \\
\text { communication improved at } \\
\text { hospitals. Course impact was noted } \\
\text { to be reduced at health centers with } \\
\text { limited staff and resources. The } \\
\text { workshops cost USD } 700-1000 \text {, } \\
\text { not including initial USD } 2000 \text { for } \\
\text { equipment }\end{array}$ \\
\hline $\begin{array}{l}\text { O’Hara, } \\
2015 \text { and } \\
\text { O’Brien, } \\
2018 \\
{[57,58]}\end{array}$ & Uganda & $\begin{array}{l}\text { Uganda Sustainable Trauma } \\
\text { Orthopedic Program (USTOP) }\end{array}$ & $\begin{array}{l}\text { Physicians, } \\
\text { Surgeons, } \\
\text { Nurses and } \\
\text { Physiotherapists }\end{array}$ & $\begin{array}{l}\text { USTOP was declared beneficial for } \\
\text { all participants and made a positive } \\
\text { impact on the surgical care of } \\
\text { patients with orthopedic trauma } \\
\text { injuries }\end{array}$ \\
\hline $\begin{array}{l}\text { Anderson, } \\
2018[59]\end{array}$ & Uganda & $\begin{array}{l}\text { "Surgical Techniques and Repairs in } \\
\text { Trauma for the Low-resource } \\
\text { Environment" (STaRTLE) and } \\
\text { "Emergency Ward Management of } \\
\text { Trauma" (EWMT) }\end{array}$ & $\begin{array}{l}\text { Residents } \\
\quad(\mathrm{STaRTLE}=8 ; \\
\quad \text { EWMT }=15)\end{array}$ & $\begin{array}{l}\text { Average trauma management } \\
\text { knowledge and understanding } \\
\text { scores improved post-training by } \\
19 \% \text { and } 23 \% \text { for EWMT and } \\
\text { STaRTLE participants, } \\
\text { respectively. Additionally, there } \\
\text { was high participant satisfaction } \\
\text { across all aspects of the course } \\
\text { (median 5/5 ratings) }\end{array}$ \\
\hline
\end{tabular}


Table 1 continued

\begin{tabular}{|c|c|c|c|c|}
\hline $\begin{array}{l}\text { First author, } \\
\text { year }\end{array}$ & Country & Program & Participants (n) & Outcome(s) \\
\hline $\begin{array}{c}\text { Ullrich, } 2020 \\
{[60,61]}\end{array}$ & Uganda & $\begin{array}{l}\text { Mulago Operative Trauma } \\
\text { Resuscitation (M-OTR) Course / } \\
\text { The Kampala Advanced Trauma } \\
\text { Course (KATC) }\end{array}$ & Residents (28) & $\begin{array}{l}\text { Although participants had } \\
\text { significantly increased post-course } \\
\text { versus pre-course test scores } \\
(\mathrm{p}<0.05), \text { there was no } \\
\text { significant increase in taught } \\
\text { trauma operative management } \\
\text { techniques }\end{array}$ \\
\hline $\begin{array}{l}\text { Edwards, } \\
2011 \text { [62] }\end{array}$ & Zambia & $\begin{array}{l}\text { American Burn Association/ } \\
\text { Children's Burn Foundation } \\
\text { (ABA/CBF) Team Training }\end{array}$ & $\begin{array}{l}\text { Healthcare } \\
\text { providers }\end{array}$ & $\begin{array}{l}\text { There was no significant } \\
\text { improvement in burn management } \\
\text { parameters (surgical intervention, } \\
\text { fluid resuscitation, skin grafting) } \\
\text { from pre-course to post-course. } \\
\text { Overall, any changes in patient } \\
\text { management did not translate into } \\
\text { improved patient outcomes }\end{array}$ \\
\hline $\begin{array}{l}\text { Tuggle, } 2017 \\
\text { [63] }\end{array}$ & Zimbabwe & $\begin{array}{l}\text { Advanced Trauma Care for Nurses } \\
\text { (ATCN) }\end{array}$ & Nurses (64) & $\begin{array}{l}\text { The study describes the first } \\
\text { successful and sustainable } \\
\text { implementation of ATCN/ATLS at } \\
\text { a nursing college in Bulawayo }\end{array}$ \\
\hline
\end{tabular}

\section{Sustainability}

In total, $4 / 45(8.9 \%)$ studies commented on course costs $[14,21,55,56]$, of which only one study examined costeffectiveness of the course teachings versus traditional care [21]. The TTT workshops in Uganda were the most inexpensive, costing \$700-1,000 USD/course, after an initial $\$ 2,000$ USD was spent on equipment [56]. The Surgical Management and Reconstructive Training orthopedic trauma course in Tanzania was more expensive, with an annual cost of \$18,000-25,000 USD [55]. A Mongolian ATLS study emphasized the benefit of training local staff as instructors, as annual course costs decreased from $\$ 68,038$ USD to $\$ 10,709$ USD when Mongolian trainers were used [14]. Carlson et al. [21] were the only study to report on the cost-effectiveness of their Orthopedic Trauma Care Specialist program, which resulted in cost savings of \$134 USD per DALY per resident trained as an orthopedic trauma specialist surgeon.

In total, five studies followed participant outcomes for a period of at least six months, with only one study following up for greater than one year [45]. No studies described a recertification process. The two studies comparing retention of knowledge several months after course administration reported retained competencies in trauma knowledge and skills at nine months [49] and two years [45]. None of the studies examined long-term patient mortality and morbidity for a period greater than one year, nor compared results to pre-course parameters.

\section{Postgraduate medical education programs}

In total, 51/79 (64.6\%) of LMICs have a form of surgical PGME program, of which 34 (66.7\%) define trauma skills as required curricular competencies (Fig. 2). These countries have formalized PGME programs in the form of residencies, fellowships, and Masters and/or Doctorates. Traumatology is most often a component of general surgery or orthopedic surgery PGME programs (Online Resource 3). Other PGME programs with required trauma competencies include neurosurgery, cardiothoracic surgery, pediatric surgery, maxillofacial surgery, urology, otorhinolaryngology, and plastic surgery. Most PGME programs are two to five years long, but time devoted to trauma training varies greatly between programs.

Several countries belong to an overarching professional society that regulates PGRME programs. In sub-Saharan Africa, this includes the College of Surgeons of East, Central, and Southern Africa (COSECSA) [8] and the West African College of Surgeons (WACS) [64]. The Arab Boards also regulate and accredit PGME programs in the Middle East, South Asia, and North Africa [65]. Although the American College of Surgeons (ACS) does not oversee PGME programs in LMICs, they do provide continuing medical education (CME) opportunities for trainees. 


\section{Discussion}

To our knowledge, this is the first review to provide a comprehensive overview of trauma training in LMICs, including both trauma courses and PGME programs. Overall, we discovered several attempts to implement various trauma training courses in LMICs, but there is little and conflicting evidence concerning the effectiveness, suitability, and sustainability of these courses long-term (Fig. 3).

\section{Inadequate needs assessments}

Most of the studies attempted to engage locals when implementing trauma courses. However, only 15 studies conducted a formal needs assessment with their target populations. Successful trauma systems in LMICs include predevelopment needs assessments that identify existing resources and serve as a foundation for future developments [66]. Future studies should include formal needs assessments with stakeholders and may include methods such as the literature reviews, community surveys, focus groups, cross-sectional observations, chart reviews, or a combination [66]. However, although formal needs assessments were not conducted, several of the courses likely enshrined local engagement into their processes through longitudinal interactions with these populations and partnerships with LMIC colleagues.

\section{Lack of course standardization}

Although many LMICs have PGME programs with trauma management competencies, the distribution, duration, and structure of these programs vary across countries and regions. These inconsistencies are further compounded by some countries belonging to multiple PGME regulatory organizations, resulting in different standards of accreditation for trainees in different specialties or hospitals across a given country. A cohesive and sustainable approach to trauma training, either regionally, nationally, or inter-regionally, has the opportunity to reduce costs and improve standardization and quality of education for HCPs involved in trauma care management.

\section{Indeterminate "best" trauma course}

A reason for the lack of guidance on mandatory trauma courses may stem from uncertainty as to the most beneficial trauma course for LMICs. Contributing to the uncertainty in course superiority is the fact that studies have failed to demonstrate equal or improved care by alternative courses in LMICs compared to ATLS, the current gold standard for trauma training in HICs [67]. The fact that the two non-ATLS courses reporting on changes in patient mortality revealed no significant difference in pre-course and post-course patient outcomes creates a question to the utility and validity of these courses in trauma education [19, 62].

ATLS was the only program to demonstrate concrete mortality benefits to injured patients; however, ATLS lacks a multidisciplinary approach that reflects the reality in LMICs. Rural Trauma Team Development Course (RTTDC) is also offered by the ACS and provides multidisciplinary trauma training and emphasizes teamwork and crisis resource management and has shown success in improving outcomes in rural settings [68]. Only one study looked at RTTDC implementation and did not link course participation to patient outcomes. However, a hybrid model of RTTDC and ATLS combining the multidisciplinary and resource management components of RTTDC with the high standards and quality of training of ATLS may be beneficial in these regions.

The lack of cost-effectiveness data of courses further adds to the ambiguity surrounding their appropriateness in an LMIC setting. Several previous studies have claimed the expensive nature of ATLS, along with reliance on highincome equipment, and make the course unsuitable for use in LMICs [52]. However, we have found a lack of evidence to support the cost-effectiveness of other trauma training courses in LMICs. Evidently, a robust health economics evaluation of the different programs, models of training, resource utilization, and patient outcomes are needed before conclusive statements on course cost-effectiveness can be made.

Overall, limitations in course evaluation with respect to cost-effectiveness and clinical outcomes can be partially attributed to the insufficient follow-up time of the studies. An essential component to CME is the process of recertification, something enshrined in the ATLS model and recommended in the WHO's Guidelines for trauma quality improvement programs [69]. None of the included studies mentioned a recertification process for participants after taking a stand-alone trauma course. This brings up an issue of sustainability and ethical responsibility to ensure trainees have opportunities to refresh their knowledge and skills, representing an area of needed improvement in trauma training in these regions. There is a substantial lack of funding for global surgery initiatives, and many of these courses are paid for through donations. A stronger global surgery funding network would bolster the scientific rigor of this subject and allow for improved outcome measurements, including longer follow-up periods. 


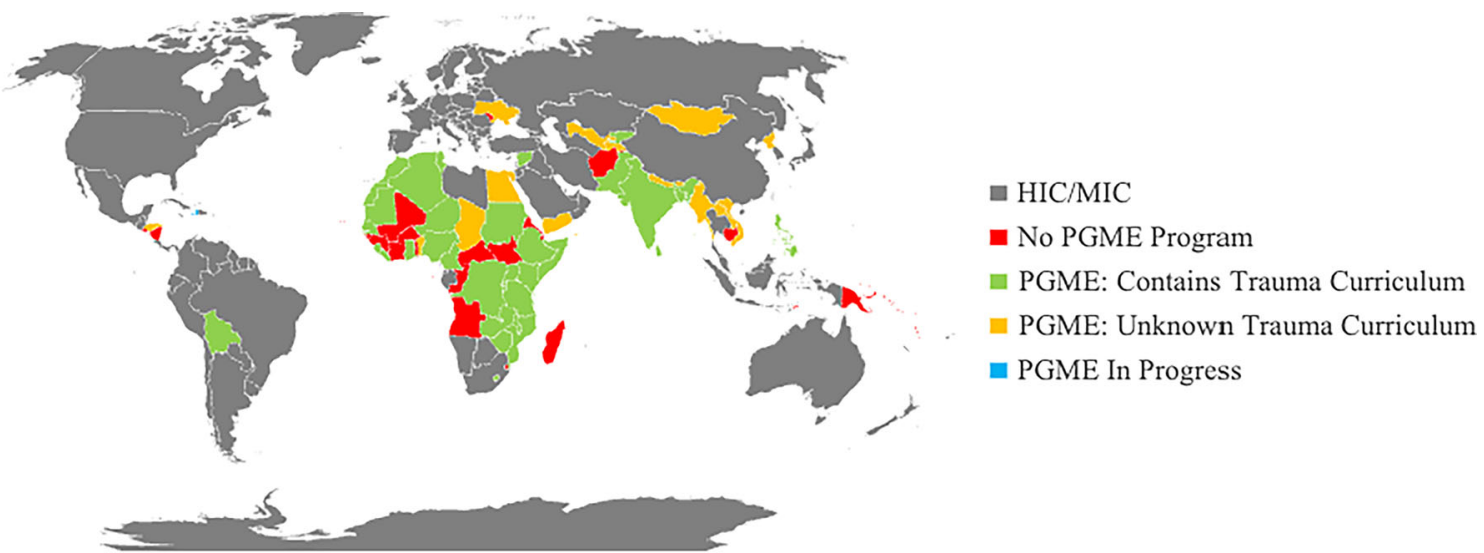

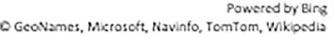

Fig. 2 Distribution of surgical PGME programs and included trauma management competencies in low- and lower middle-income countries. Country income group determined by the World Bank Report,

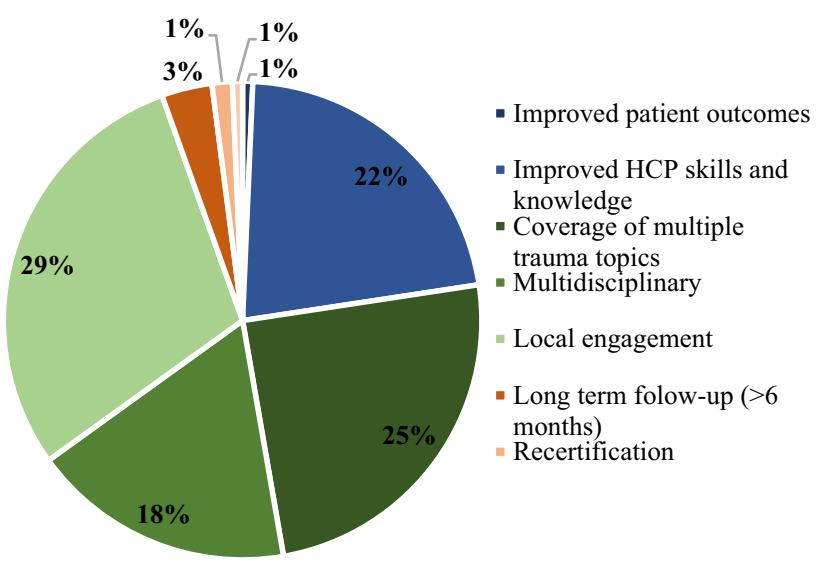

Fig. 3 Number of courses demonstrating improvements in effectiveness, suitability, and sustainability of trauma training. Blue, effectiveness categories; green, suitability categories; and orange, sustainability categories

\section{Limitations}

These findings should be interpreted with respect to limitations of this study. This review was only able to capture information on trauma programs published online. Furthermore, the search strategy was conducted in English and therefore may have missed articles or websites in different languages. Consequently, PGME programs may exist that were not identified. Additionally, available websites for PGME programs may not be up to date or reflect actual functioning of the programs.
2020. HIC, high-income country; MIC, middle-income country (including upper middle income); PGME, postgraduate medical education

NGOs such as Médecins Sans Frontières and the International Committee of the Red Cross train HCPs during their deployments in trauma care. These trainings are not typically published as research articles and traditionally rely on HCPs from HICs and therefore were outside of the scope of this review. However, these organizations are increasingly providing multidisciplinary trauma training to local practitioners and are important collaborators and funders of trauma training in LMICs.

Furthermore, we excluded laypeople trained in trauma care. Laypeople play an important role in trauma management in LMICs, especially in rural and pre-hospital settings [70]. However, in an attempt to succinctly define parameters of this study, we chose to focus exclusively on trained HCPs providing definitive trauma care. This narrowed the scope of our results, providing a reductionist picture of the trauma care as a whole in LMICs.

\section{Conclusion}

This scoping review is the first known study to provide a comprehensive examination of trauma training opportunities, including PGME programs in LMICs. Overall, this study has illuminated the notable lack of standardization and evidence-based implementation of trauma training in these regions. It highlights the fragmentation in trauma training in many LMICs when well-intentioned players, often from HICs, introduce multiple courses without needsinformed engagement from local stakeholders. Although several different trauma courses have been executed in LMICs, studies have failed to prove long-term mortality, 
Fig. 4 Summary of recommendations
Trauma Care Training Courses:

- Need for a standardization of trauma course offerings across a country and/or region

- Before implementing a course, studies should include a formal needs assessment with local stakeholders

- Courses should be modified from high-income models to fit the sociocultural, geographic, and economic contexts of target LMICs

- All courses should demonstrate sustainability in the setting into which they are implemented and show key outcome measures such as mortality and morbidity benefits and cost-effectiveness

- A maintenance or recertification process should be in place for participants of courses

- Courses should be implemented into a larger, cohesive trauma systems' development plan

Post Graduate Medical Education Programs:

- All General Surgery and Orthopedic Surgery residency programs should include trauma management competencies

- All surgical residents should complete a trauma care training course and be routinely recertified in the course as part of their CME morbidity, and/or economic benefits. Trauma training programs should be required to demonstrate these outcomes and establish a standardized and longitudinal process for HCP trauma certification in a given country or region. A comprehensive and effective trauma education program should include short courses like ATLS that are contextualized to the setting, incorporated into CME, and demonstrate tangible benefits to patients and the healthcare system (Fig. 4).

Authors' contributions RL, AS, and HGH designed the study. JK designed the search strategy and helped with methods. RL, XC, and SD performed the literature review and study selection. RL performed data analysis and wrote the article. AS, HGH, EJ, XC, and SD served as additional reviewers, made substantial contributions to the discussion, and critically revised the article for content. All authors read and improved the final article.

Funding The authors declare no funding.

\section{Declarations}

Conflict of interest The authors declare that they have no competing interests to disclose.

Supplementary Information The online version contains supplementary material available at https://doi.org/10.1007/s00268021-06283-1.

Open Access This article is licensed under a Creative Commons Attribution 4.0 International License, which permits use, sharing, adaptation, distribution and reproduction in any medium or format, as long as you give appropriate credit to the original author(s) and the source, provide a link to the Creative Commons licence, and indicate if changes were made. The images or other third party material in this article are included in the article's Creative Commons licence, unless indicated otherwise in a credit line to the material. If material is not included in the article's Creative Commons licence and your intended use is not permitted by statutory regulation or exceeds the permitted use, you will need to obtain permission directly from the copyright holder. To view a copy of this licence, visit http://creativecommons. org/licenses/by/4.0/.

\section{References}

1. Haagsma JA, Graetz N, Bolliger I et al (2015) The global burden of injury: incidence, mortality, disability-adjusted life years and time trends from the Global Burden of Disease study 2013. Inj Prev 22:3

2. Murray CJL, Ortblad KF, Guinovart C et al (2014) Global, regional, and national incidence and mortality for HIV, tuberculosis, and malaria during 1990-2013: a systematic analysis for the global burden of disease study 2013. Lancet 384:1005-1070

3. Stewart BT, Quansah R, Gyedu A et al (2016) Serial assessment of trauma care capacity in Ghana in 2004 and 2014. JAMA Surg 151:164-171

4. Sethi D, Aljunid S, Sulong SB, Zwi AB (2000) Injury care in low- and middle-income countries: identifying potential for change. Inj Contr Saf Promot 7(3):153-164

5. Drimousis PG, Theodorou D, Toutouzas K et al (2011) Advanced Trauma Life Support certified physicians in a non trauma system setting: Is it enough? Resuscitation 82(2):180-184

6. Ley Greaves RA, Wilkinson LF, Wilkinson DA (2017) Primary Trauma Care: a 20-year review. Trop Doct 47(4):291-294

7. Jawaid M, Ahmed Memon A, Masood Z, Nadeem Alam S (2013) Effectiveness of the Primary Trauma Care course: Is the outcome satisfactory? Pak J Med Sci 29(5):1265-1268

8. The College of Surgeons of East, Central and Southern Africa (2017) Training curriculum: fellowship in general surgeryFCSgen (ECSA). http://membership.cosecsa.org/wp-content/ uploads/2019/09/COSECSA-Overall-Curriculum.pdf

9. McCullough C, DeGennaro V, Bagley JK et al (2016) A national trauma capacity assessment of Haiti. J Surg Res 201:126-133

10. Tricco AC, Lillie E, Zarin W, O'Brien KK et al (2018) PRISMA Extension for Scoping Reviews (PRISMAScR): Checklist and Explanation. Ann Intern Med 169:467-473

11. Mock C, Lormand JD, Goosen J et al (2004) Guidelines for essential trauma care. World Health Organization, Geneva

12. Van Heng Y, Davoung C, Husum H (2008) Non-doctors as trauma surgeons? A controlled study of trauma training for nongraduate surgeons in rural Cambodia. Prehosp Disaster Med $23: 483-489$ 
13. Tajsic N, Sambath P, Nguon S et al (2017) Open fracture management in low-resource settings: a medical training experience in Cambodian hospitals. World J Surg 41:2981-2989. https://doi. org/10.1007/s00268-017-4245-7

14. Kornfeld JE, Katz MG, Cardinal JR et al (2018) Cost analysis of the Mongolian ATLS@ Program: a framework for low- and middle-income countries. World J Surg 43:353. https://doi.org/ 10.1007/s00268-018-4795-3

15. Richard AJ, Lee CI, Richard MG et al (2009) Essential trauma management training: addressing service delivery needs in active conflict zones in eastern Myanmar. Hum Resour Health 7:19

16. Yu DTY, Gillon JT, Dickson R et al (2017) Developing a sustainable need-based pediatric acute care training curriculum in Solomon Islands. Front Public Health 5:86

17. Lam NN, Huong HTX, Tuan CA (2018) Preparation for major burns incidents: evaluation of continuing medical education training courses for professionals. Ann Burns Fire Disasters 31(4):322-328

18. Choi S, Kim J, Heo J et al (2020) Outcomes of trauma education workshop in Vietnam: improving diagnostic and surgical skills. BMC Med Educ 20:1-267

19. Cioè-Peña E, Granados J, Wrightsmith L et al (2017) Development and implementation of a hospital-based trauma response system in an urban hospital in San Salvador, El Salvador. Trauma 19:118-126

20. Dickason RM, Cioè-Peña E, Chisolm-Straker M (2017) Primary Trauma Care curriculum: a qualitative analysis of impediments to improvement. Trauma 19:127-132

21. Carlson LC, Slobogean GP, Pollak AN (2012) Orthopaedic trauma care in Haiti: a cost-effectiveness analysis of an innovative surgical residency program. Value Health 15:887-893

22. Normore R, Greene H, DeLong A, Furey A (2017) The Orthopedic Trauma Symposium: improving care of orthopedic injuries in Haiti. Can J Surg 60:228-235

23. Kurdin A, Caines A, Boone D, Furey A (2018) TEAM: a low-cost alternative to ATLS for providing trauma care teaching in Haiti. J Surg Educ 75:377-382

24. Pringle K, Mackey JM, Modi P et al (2015) A short trauma course for physicians in a resource-limited setting: Is low-cost simulation effective? Injury 46:1796-1800

25. El-Shinawi M, McCunn M, Sisley AC et al (2015) Developing sustainable trauma care education in Egypt: sequential Trauma Education Program, STEPs to success. J Surg Educ 72:e29-e32

26. Alwawi A, Amro N, Inkaya B (2019) The effectiveness of the Primary Trauma Care Courses in West Bank, Palestine: Are the outcomes acceptable? J Educ and Pract 10(9):105-109

27. Biswas A, Rahman F, Pm M et al (2017) An evaluation of Emergency Management of Severe Burn (EMSB) course in Bangladesh: a strategic direction. Burns Trauma 5:12

28. Tchorz KM, Thomas N, Jesudassan S et al (2007) Teaching trauma care in India: an educational pilot study from Bangalore. J Surg Res 142:373-377

29. Light TD, Latenser BA, Heinle JA et al (2009) The partnership of the American Burn Association, Children's Burn Foundation, and the Pediatric Burn Team in Vellore, India-a progress report. J Burn Care Res 30(1):46-49

30. Douglas R, Vasanthi B, Giles A, Kumar G (2010) Improving trauma care in India: a recommendation for the implementation of ATLS training for emergency department medical officers. Int J Emerg Med 3:27-32

31. Ali J, Kumar S, Gautam S et al (2015) Improving trauma care in India: the potential role of the Rural Trauma Team Development Course (RTTDC). Indian J Surg 77:227-231

32. O'Reilly G, Fitzgerald M, Ariyananda PL et al (2009) Trauma reception and resuscitation in Sri Lanka: the health for the south Capacity-Building project. Emerg Med Australas 21:147-152
33. O'Reilly GM, Fitzgerald M, Dewan Y et al (2011) The Alfred trauma team training program in India and Sri Lanka. Emerg Med Australas 23:632-639

34. Shrestha R, Khadka SK, Thapa S et al (2018) Improving knowledge, skill and confidence of novice medical doctors in trauma management with principles of ABCDE. Kathmandu Univ J Med 16:69-73

35. Jawaid M, Memon AA, Masood Z, Alam SN (2013) Effectiveness of the primary trauma care course: Is the outcome satisfactory? Pak J Med Sci 29:1265-1268

36. Sadiq MA, Rehman KU, Tariq N, Bashir EA (2018) Impact of Primary Trauma Care workshop on the cognitive domain of final year medical students. J Surg Pakistan 23(2):64-67

37. Soomro R, Ali S (2020) Trauma Evaluation and Management (TEAM) course for medical students in Pakistan. Pak J Med Sci 36:1257-1262

38. Ologunde R, Le G, Turner $\mathbf{J}$ et al (2017) Do trauma courses change practice? A qualitative review of 20 courses in east, central and southern Africa. Injury 48:2010-2016

39. Peter NA, Pandit H, Le G et al (2015) Delivering a sustainable trauma management training programme tailored for low-resource settings in east, central and southern African countries using a cascading course model. Injury 47:1128-1134

40. Nogaro M, Pandit H, Peter N et al (2015) How useful are primary trauma care courses in sub-Saharan Africa? Injury 46:1293-1298

41. Macleod JBA, Gravelin S, Jones T et al (2009) Assessment of acute trauma care training in Kenya. Am Surg 75(11):1118-1123

42. MacLeod J, Okech M, Labib M et al (2011) Evaluation of trauma and critical care training courses on the knowledge and confidence of participants in Kenya and Aambia. World J Surg 35:9-16. https://doi.org/10.1007/s00268-010-0810-z

43. O'Sullivan J, O'Connor G (2012) Global emergency care skills: Does it work? African J Emerg Med 2:136-140

44. Shaye DA, Tollefson T, Shah I et al (2018) Backward planning a craniomaxillofacial trauma curriculum for the surgical workforce in low-resource settings. World J Surg 42:3514. https://doi.org/ 10.1007/s00268-018-4690-y

45. Tolppa T, Vangu A, Balu H et al (2020) Impact of the primary trauma care course in the Kongo Central Province of the Democratic Republic of Congo over two years. Injury $51: 235-242$

46. Mock CN, Quansah R, Addae-Mensah L, Donkor P (2005) The development of continuing education for trauma care in an African nation. Injury 36:725-732

47. Quansah R, Abantanga F, Donkor P (2008) Trauma training for non-orthopaedic doctors in low- and middle-income countries. Clin Orthop Relat Res 466:2403-2412

48. Berndtson AE, Morna M, Debrah S et al (2019) The TEAM (Trauma Evaluation and Management) course: medical student knowledge gains and retention in the USA versus Ghana. Trauma Surg Acute Care Open 4:e000287

49. Wanjiku G, Janeway H, Foggle J et al (2017) Assessing the impact of an emergency trauma course for senior medical students in Kenya. African J Emerg Med 7:167-171

50. Hill KA, Johnson ED, Lutomia M et al (2018) Implementing the Trauma Evaluation and Management (TEAM) course in Kenya. J Surg Res 232:107-112

51. Young S, Banza L, Mkandawire N (2016) The impact of long term institutional collaboration in surgical training on trauma care in Malawi. Springerplus 5:407

52. Petroze RT, Byiringiro JC, Ntakiyiruta G et al (2015) Can focused trauma education initiatives reduce mortality or improve resource utilization in a low-resource setting? World J Surg 39:926-933. https://doi.org/10.1007/s00268-014-2899-y 
53. Bergman S, Deckelbaum D, Lett R et al (2008) Assessing the impact of the Trauma Team Training program in Tanzania. J Trauma 65:879-883

54. Mitchell KB, Giiti G, Kotecha V et al (2013) Surgical education at Weill Bugando Medical Centre: Supplementing surgical training and investing in local health care providers. Can J Surg 56:199-203

55. Carey J, Caldwell A, Coughlin R, Hansen S (2015) Building orthopaedic trauma capacity: IGOT international SMART course. J Ortho Trauma 29(Suppl 10):S17-S19

56. Lett R, Kobusingye O, Asingwire N, Ssengooba F (2004) Trauma Team Training course: evaluation of Ugandan implementation. Afr Saf Promot 2:78-82

57. O'Hara NN, O'Brien PJ, Blachut P (2015) Developing orthopaedic trauma capacity in Uganda. J Ortho Trauma 29:S20-S22

58. O'Brien P, Kajja I, Potter JM et al (2018) Role of North-South partnership in trauma management: Uganda Sustainable Trauma Orthopaedic Program. J Ortho Trauma 32:S21-S24

59. Anderson GA, Kayima P, Ilcisin L et al (2018) Development of a comprehensive trauma training curriculum for the resource-limited environment. J Surg Educ 75:1317-1324

60. Ullrich SJ, Kilyewala C, Lipnick MS et al (2020) Design, implementation and long-term follow-up of a context specific trauma training course in Uganda: Lessons learned and future directions. Am J Surg 219:263-268

61. Ullrich SJ, DeWane MP, Cheung M et al (2020) Development of an operative trauma course in Uganda-a report of a three-year experience. J Surg Res 256:520-527

62. Edwards D, Heard J, Latenser BA et al (2011) Burn injuries in Eastern Zambia: impact of multidisciplinary teaching teams. J Burn Care Res 32(1):31-38
63. Tuggle D, Rasnake N, Day T (2017) Improving trauma care in Africa. J Trauma Nurs 24:258-260

64. West African College of Surgeons (n.d.) Faculty of Surgery Training Programs and Curricula. https://www.wacscoac.org/ downloads/SURGERY\%20CURRICULUM.pdf

65. The Arab Boards (2020) http://arab-board.org/en/content/generalsurgery

66. Callese TE, Richards CT, Shaw P et al (2015) Trauma system development in low- and middle-income countries: a review. J Surg Res 193(1):300-307

67. Carmont MR (2005) The Advanced Trauma Life Support course: a history of its development and review of related literature. Postgrad Med J 81(952):87-91

68. Vella MA, Sikoutris S, Chreiman K et al (2019) Longitudinal experience with RTTDC: improving outcomes through collaboration. ACS Bull 104:22-30

69. Mock C, Juilliard C, Brundage S, Goosen J, Joshipura M (2009) World Health Organization: guidelines for trauma quality improvement programs. https://apps.who.int/iris/bitstream/han dle/10665/44061/9789241597746_eng.pdf;jsessionid=3A0FF532 EE11BE90A69259940EDB4B70? sequence $=1$

70. Callese TE, Richards CT, Shaw P et al (2014) Layperson trauma training in low- and middle-income countries: a review. J Surg Res 190(1):104-110

Publisher's Note Springer Nature remains neutral with regard to jurisdictional claims in published maps and institutional affiliations. 